\title{
Non-invasive quantification of aortic regurgitation by Doppler echocardiography
}

\author{
B DIEBOLD, P PERONNEAU, „ D BLANCHARD, G COLONNA, J L GUERMONPREZ, \\ J FORMAN, P SELLIER, P MAURICE
}

From the Clinique Cardiologique and ERA-CNRS 785; Hôpital Broussais, Paris, France

SUMMARY This study was undertaken to assess the contribution of Doppler echocardiography to the quantification of aortic valve regurgitation. Ultrasound examination was performed by recording aortic arch blood flow from the suprasternal notch. A non-invasive index of valve regurgitation was obtained by calculating the ratio between the maximal amplitude of forward flow during systole and the amplitude of retrograde flow during diastole measured at the onset of the $R$ wave of the electrocardiogram. This index was compared with semiquantitative data derived from supravalvular aortography in 93 patients. In pure aortic regurgitation (67 patients) the results showed a high correlation coefficient between Doppler and angiographic estimates. In cases of associated aortic valve stenosis there were problems in the accurate estimation of systolic blood flow which led to global overestimation in general of the degree of regurgitation and considerable lack of precision in individual patients. But in general Doppler echocardiography appeared to be a successful technique to quantify pure aortic regurgitation.

Quantification of aortic valve regurgitation is valuable not only in deciding on surgical intervention but also in planning mitral valve surgery when associated aortic reflux may cause flooding of the operative field. Clinical evaluation of aortic regurgitation is unreliable except in cases where aortic reflux is torrential. Among non-invasive methods of assessment, echocardiography provides only indirect pointers to the diagnosis which may become completely valueless when significant mitral valve disease or severe left ventricular impairment with cavity dilatation are associated. This technique does not allow aortic reflux to be quantified in a reliable fashion. ${ }^{1}$ Supravalvular aortography is still the most widely used method to quantify aortic regurgitation even though this procedure is invasive and requires arterial cannulation.

Doppler echocardiography and conventional echocardiography are non-invasive techniques. The ability to measure flow velocities at different depths has made this investigation particularly suitable for cardiological use. The technique has already been suggested for the evaluation of aortocoronary bypass graft patency, ${ }^{2}$ mitral stenosis, ${ }^{34}$ mitral regurgita-

\footnotetext{
*Maitre de recherche with INSERM.
}

Accepted for publication 21 September 1982 tion, ${ }^{56}$ ventricular septal defect, ${ }^{78}$ atrial septal defect, ${ }^{9}$ pulmonary hypertension and persistent ductus arteriosus, ${ }^{10}$ aortic stenosis, ${ }^{11}$ and aortic regurgitation. ${ }^{12-14}$ The present study compares the degree of aortic regurgitation estimated by supravalvular aortography with quantitative measurements derived from Doppler echocardiography.

\section{Subjects and methods}

\section{PRINCIPLES}

Doppler echocardiography has been adapted to the study of blood flow from techniques developed in radar systems. ${ }^{2-4}$ Curves are obtained by comparing the frequencies of emitted and back scattered ultrasound. These curves are related to the velocity of red cells by a factor that depends in particular on the angle between the ultrasound beam and the axis along which the target cells are moving. Pulsed emission provides echocardiographic data and allows selection of the depth and thickness of the sample volume.

The equipment used in this report was developed by ERA-CNRS 785 (Paris, France) and details have previously been published. ${ }^{246}$ Its main features are summarised in the Table.

The Doppler signal is processed by a zero crossing 
Table Main features of the apparatus

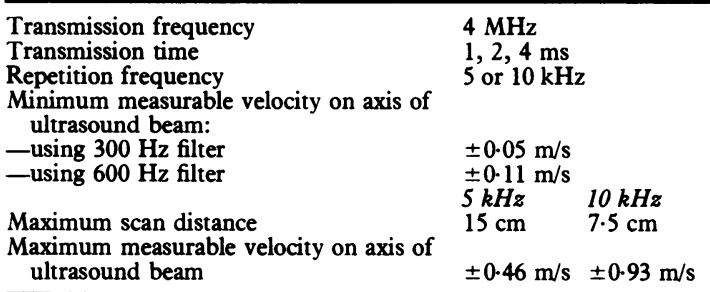

counter and an integrator which produces an analogue curve.

\section{TECHNIQUE OF PATIENT EXAMINATION (Fig. 1)}

The patient is placed in a supine position. The transducer is positioned in the suprasternal notch and directed downwards, backwards, and to the left in order to bring the ultrasound beam on to the thoracic aorta between its horizontal and descending portions. Blood flow in this segment of the aorta is recognised by two features, namely that flow is recorded from between two walls of a large calibre vessel and that it moves away from the sample volume during systole (Fig. 2). In this position blood flow in the subclavian artery moves toward the transducer during systole

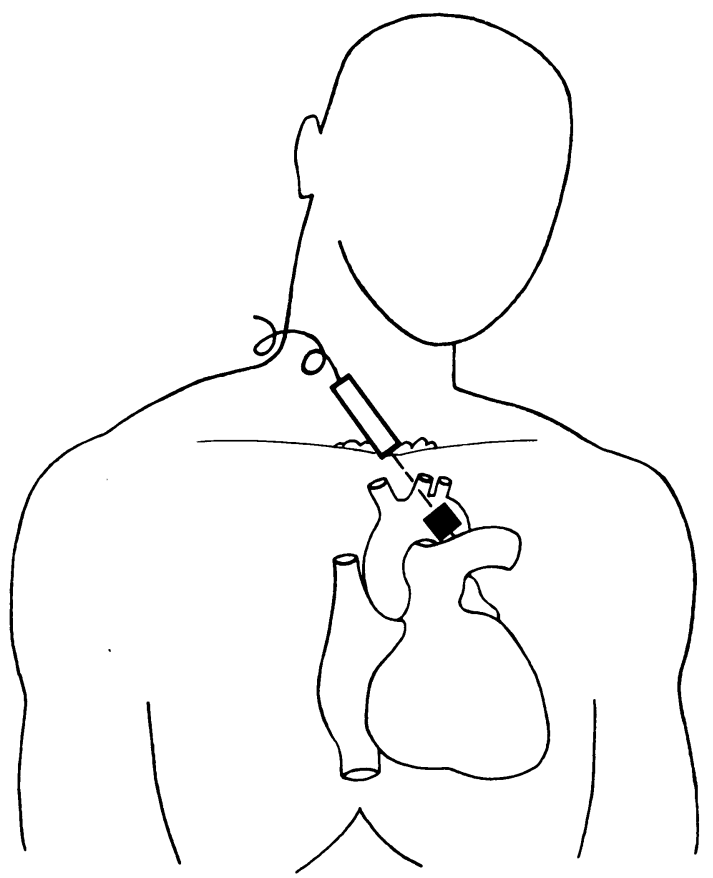

Fig. 1 Illustration of the suprasternal approach for recording aortic arch blood flow.

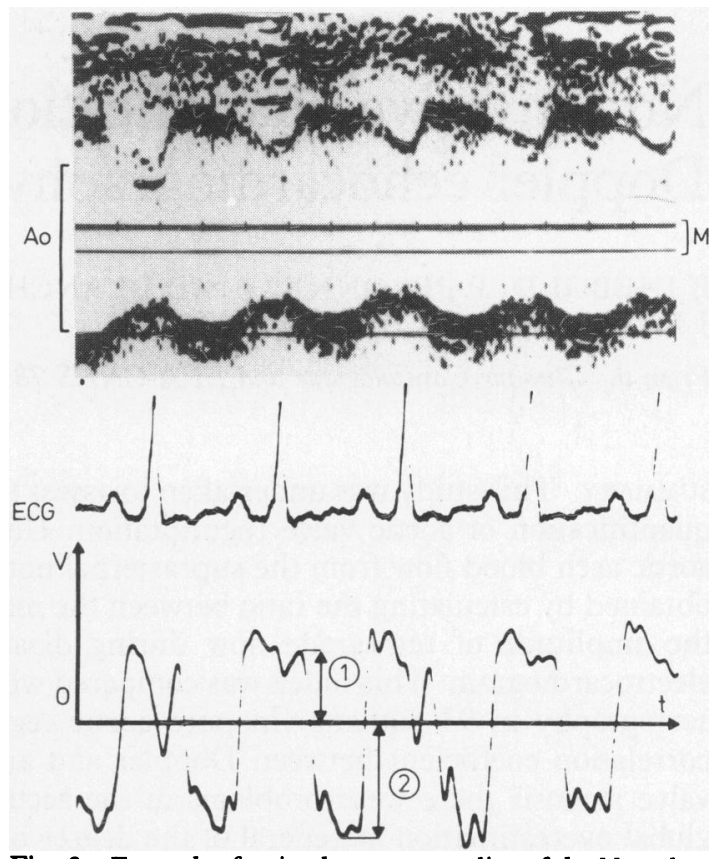

Fig. 2 Example of a simultaneous recording of the $M$ mode echo and of the mean velocity curve during an examination of the aorta from the suprasternal notch. Notice the variation of the diameter of the aorta during the cardiac cycle. $M$, measurement volume; $t$, time; 0 , zero velocity; $V$, velocity, Ao, aorta.

and ceases during late diastole; blood flow in the left $\frac{0}{3}$ common carotid artery also exhibits forward systolic movement but in addition there is a continuous forward component during diastole; blood in the subcla- $\overrightarrow{5}$ vian and jugular veins flows away from the transducer in the same direction both in systole and diastole. In $\frac{7}{\sigma}$ cases of severe tricuspid regurgitation venous blood 3 . flow may be confused with arterial flow but it can be recognised by its behaviour with respiration. Finally, $\overline{3}$ the luminal calibre of the above vessels appears much $ᄋ$ smaller than that of the aorta when judged by echocardiographic landmarks. The combination of $\frac{7}{2}$ directional changes in flow with the ability to use depth discrimination and echocardiographic reference $N$ points makes recognition of the aorta straightforward. N Nevertheless, a possible source of error may arise in $\underset{\mathrm{W}}{ }$ the aortic region, namely the origin of the left com- 0 mon carotid artery, which is characterised by anterograde diasiolic flow occurring in the same direction as systolic flow. When this feature is detected the trans- $\stackrel{D}{\rightarrow}$ ducer direction should be altered to record velocities more distally. The aorta is recognised by using the lowest repetition frequency, but aortic blood flow $\vec{\odot}$ must be recorded using the highest possible repetition $\underset{\mathbb{D}}{\mathbb{P}}$ frequency in order to avoid errors caused solely by 
aliaising (Fig. 3) (Appendix 1). If high repetition frequencies are not sufficient, the transducer position must be tilted so that the ultrasound beam is more perpendicular to the stream of flow.

\section{CURVE ANALYSIS}

The curves obtained during a Dopper examination reflect the time dependent variation of the average velocity encountered within the sample volume, and display blood flow approaching the transducer as a positive deflection. They may be described as follows.

\section{Normal flow (Fig. 4)}

This curve describes a $U$ shape that corresponds to the variation of blood flow during mechanical systole. After a transient reversal the curve returns to zero during diastole.

\section{Aortic regurgitation (Fig. 5)}

This condition is characterised by diastolic flow moving towards the transducer and persisting into late
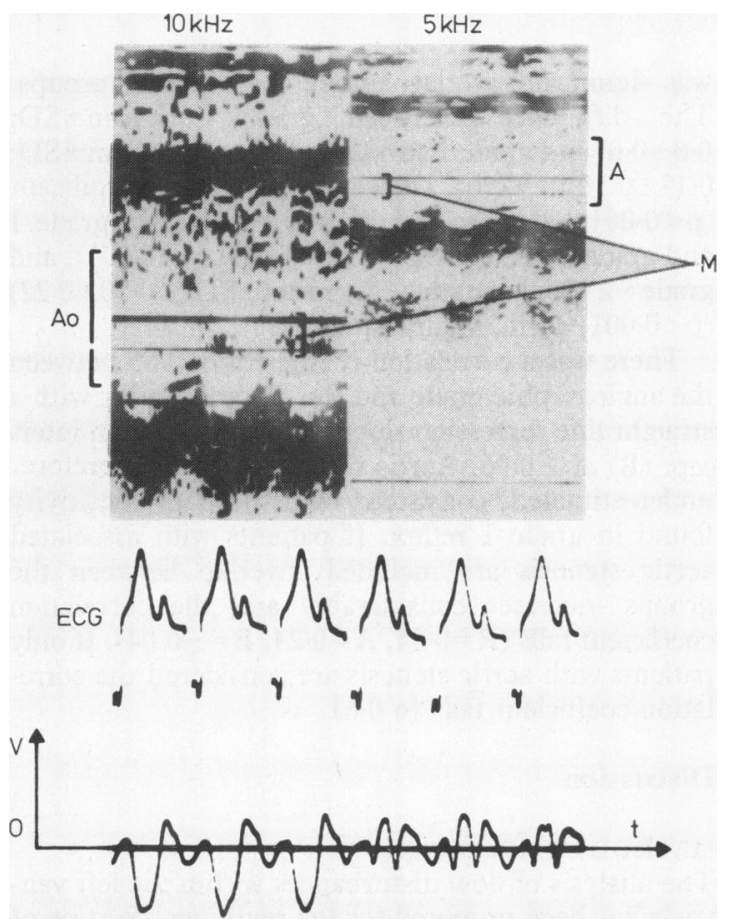

Fig. 3 Example of a velocity ambiguity. To the left, aortic flow recorded with a high pulse repetition rate $(10 \mathrm{KHz})$. To the right, the same flow recorded with the same position of the probe but with a pulse repetition rate of $5 \mathrm{KHz}$. With the high pulse repetition rate the velocities are accurately measured but the maximal depth of analysis is small. When the lower pulse repetition rate is used, the maximal depth increases but the flow is encroached by an artefact. Abbreviations as in Fig. 2.
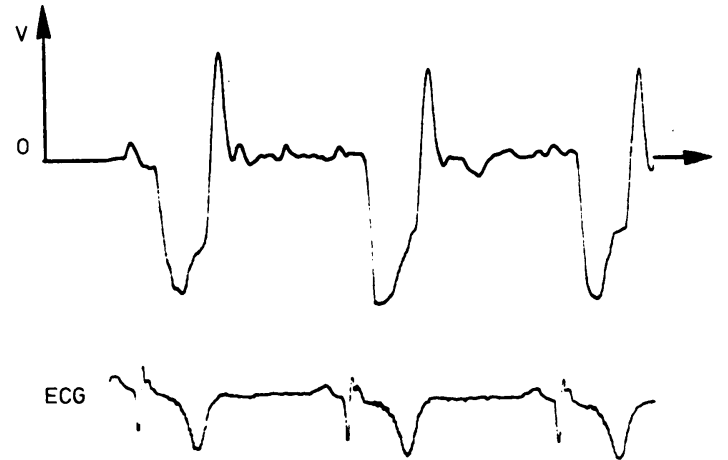

Fig. 4 Example of normal aortic flow.
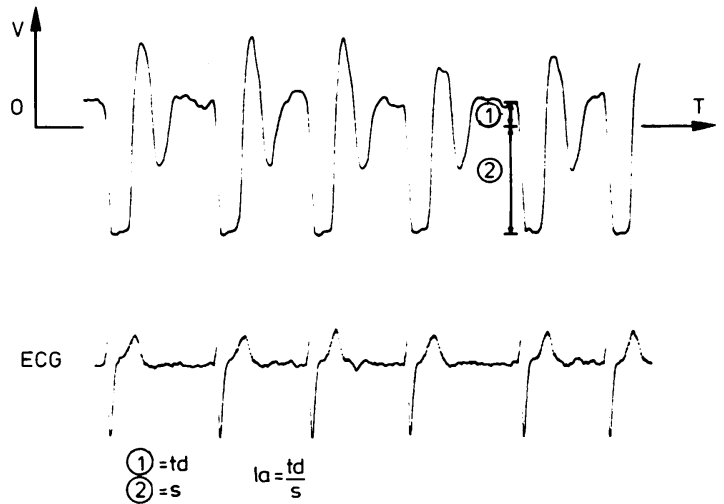

Fig. 5 Example of aortic regurgitation. $t$, velocity of the diastolic blood flow at the electrocardiographic $R$ wave; $s$, peak velocity of the systolic anterograde component. Notice that the reflected waves during the first part of diastole are particularly important in this example.

diastole. Quantification of aortic regurgitation is obtained by calculating the ratio (Ia) between the velocity of diastolic blood flow measured at the time of the electrocardiographic $\mathbf{R}$ wave (td) and the maximum systolic blood flow (S): $\mathrm{Ia}=\frac{\mathrm{td}}{\mathrm{s}}$. In this study we have used the mean value of this ratio calculated from 10 consecutive cycles.

\section{PATIENTS AND METHODS}

This study was performed on patients undergoing hospital investigation for valvular heart disease. The distribution of valve disease was as follows: 53 patients with pure aortic regurgitation, 26 patients with mixed aortic valve disease, and 14 patients without aortic regurgitation. Among these 93 patients there were 60 cases of associated mitral valve disease. There were 36 female and 57 male patients with an 
average age of 38 years (range 8 to 57 years). Doppler echocardiography was performed in all cases within 72 hours of the haemodynamic study, the operator being denied details of the history and clinical examination. Aortic regurgitation was evaluated by supravalvular aortography performed in the right anterior oblique projection using a catheter placed approximately $2 \mathrm{~cm}$ above the aortic valve and contrast medium injected at a rate of $15 \mathrm{ml} / \mathrm{s}$ for three seconds.

Radiological appearances were used to define a semiquantitative 4 point scale according to the following criteria:

Grade 0: no passage of contrast medium into the left ventricle.

Grade 1: aortic regurgitation which failed to opacify the whole cavity of the left ventricle.

Grade 2: complete opacification of the left ventricle produced only after several diastoles.

Grade 3: complete opacification of the left ventricle during the first diastole after the onset of contrast medium injection.

Comparison of ultrasound results and angiographic data was performed by calculating sensitivity and specificity, analysing statistically the differences between the groups, and calculating a correlation coefficient.

\section{Results}

Reproducibility of the curves was checked by two different operators performing measurements on 15 separate patients. Comparison of the results between Doppler echocardiographic data (ordinate) and the angiographic classification (abscissa) is shown in Fig. 6.

\section{DIAGNOSTIC VALUE}

The specificity found in this study was encouraging. In the group with grade 0 aortic regurgitation, there was no evidence of an aortic leak by Doppler examination.

In 32 patients with grade 1 aortic regurgitation Doppler echocardiography showed evidence of aortic regurgitation in 25 patients, with seven false negatives. Aortic regurgitation, however, was correctly diagnosed by ultrasound in all patients with grade 2 and 3 aortic reflux. Overall specificity and sensitivity was $100 \%$ and $92 \%$, respectively. If patients with minimal aortic regurgitation are excluded the sensitivity reaches $100 \%$.

\section{QUANTITATIVE VALUE}

If only the 67 patients without aortic stenosis are considered, the value of the Doppler index increases progressively with increasing degrees of aortic regurgitation. The standard deviation was large and there

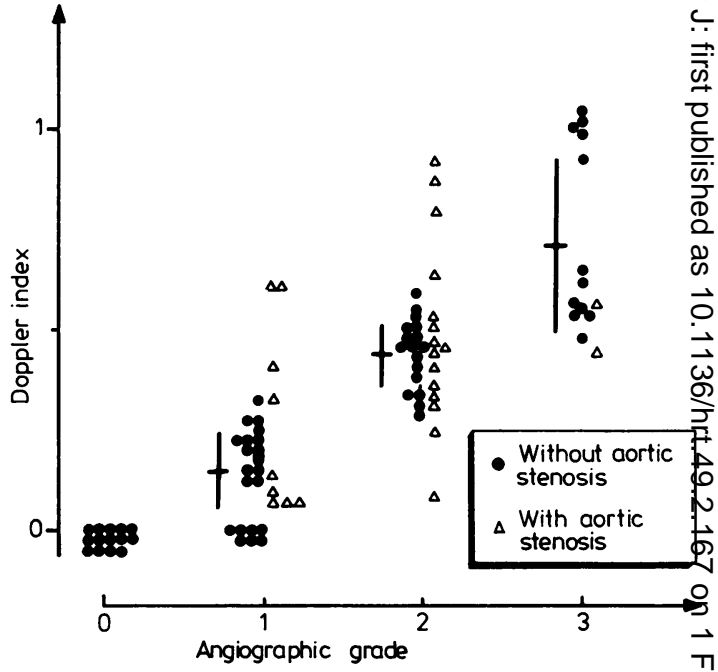

Fig. 6 Diagrammatic illustration of the comparison between $\frac{D}{O}$ Doppler echocardiography and aortography for the evaluation of aortic regurgitation.

was significant overlap among the different groups. The differences between grade 0 (mean \pm SD $0.0 \pm 0.0$ ) and grade 1 aortic regurgitation (mean $\pm \mathrm{SD}_{5}^{5}$ $0 \cdot 13 \pm 0 \cdot 10)$ were, however, highly significan $(\mathbf{p}<0.001)$, as were the differences between grade ${ }^{*}$ and grade 2 (mean \pm SD: $0.43 \pm 0.009)(\mathrm{p}<0.001)$, and grade 2 and grade 3 (mean $\pm S D: 0.70 \pm 0.22$ $(\mathrm{p}<0.001)$ aortic regurgitation.

There was a correlation coefficient of 0.89 betwee the angiographic grade and the Doppler index with straight line regression slope (A) of 0.24 and an inters cept (B) of $-\mathbf{0} 06$. Aortic regurgitation is, therefore underestimated, consistent with the poor sensitivity found in grade 1 reflux. If patients with associated. aortic stenosis are included, overlap between this groups increases considerably and the correlation coefficient falls $(R=0.81, A=0.24, B=-0.04)$. If onl\$ patients with aortic stenosis are considered the corre lation coefficient falls to 0.61 .

\section{Discussion}

\section{METHOD OF APPROACH}

The analysis of flow disturbances within the left ven tricle has been proposed for the semiquantification of aortic regurgitation ${ }^{12}$; in using this approach the ovef lap between groups seems greater than in our study and, moreover, associated mitral stenosis can lead to misinterpretation. The limitations of the parasterna approach were reported also by another group ${ }^{14}$; $\vec{f}$ needs to be emphasised that the ultrasound beam propagated along a line almost perpendicular to the 
axis of blood flow and therefore a less than satisfactory angle of projection prevails though the echographic image is of optimal quality.

Positioning of the ultrasound beam is particularly advantageous when using the suprasternal approach. In the majority of patients this approach allows detailed examination of the ascending and descending aorta provided a small, single crystal transducer is used. Blood flow studies in the ascending aorta provide an accurate diagnosis of regurgitation ${ }^{1314}$ but attempts to quantify it are unsatisfactory, probably because of disturbances of velocity distribution in this region. In contrast, velocity profiles in the descending aorta seem much flatter, chiefly because this site is situated further from the aortic orifice.

\section{CURVES}

Normal blood flow curves and those recorded in moderate aortic regurgitation correspond on average to results obtained using intravascular flowmeters. ${ }^{15}$ In contrast, in some cases of massive aortic regurgitation disturbances of systolic blood flow may be encountered even in the absence of associated aortic stenosis. In this situation the systolic waveform is modified to produce an irregular quivering appearance. Echocardiographic features of the aortic valve orifice allow the phenomenon to be recognised easily. Such alterations of systolic blood flow seem to explain in part why values of the Doppler index exceed unity. Another factor relevant to these unusual results may be systolic dilatation of the aorta, which is well shown in Fig. 2.

\section{DOPPLER ECHOCARDIOGRAPHIC INDEX}

Flow reversal, seen in normal curves at the beginning of diastole, corresponds to wave reflections of pressure and flow. This phenomenon prevents use of area methods to analyse the data curves (Fig. 5). Indeed, in aortic regurgitation it is impossible to define a sharp borderline between protodiastolic flow corresponding to compliance alterations and diastolic reflux caused by valve regurgitation. For this reason the index of quantification that we have chosen to use is the ratio between end-diastolic and maximal systolic blood flow velocities. This method minimises the problems of transducer inclination, as forward and backward systolic blood flow may be considered to occur in the same axis resulting in the same angle of incidence in both numerator and denominator, therefore allowing mutual cancellation of this factor. Moreover, the size of the measurement volumes curtails the inclusion of non-representative juxtaparietal flow.

\section{METHOD OF EVALUATION}

The use of ascending aortography requires a few words of explanation. There is no doubt that results depend upon catheter position, rate and volume of contrast medium injection, left ventricular size, and the presence of mitral regurgitation, as two recent studies have emphasised. ${ }^{16} 17$ Nevertheless its value has also been clearly shown, as long as standard projections are used. ${ }^{18-20}$ At the present time it remains the most frequently employed technique for patient evaluation in routine clinical practice.

\section{RESULTS}

\section{Diagnostic value}

The sensitivity of Doppler echocardiography is good in cases of moderate and severe aortic regurgitation. False negatives occurred only in patients with minor valve reflux. This phenomenon may be the result of factors related to ascending aortic compliance or of the use of high pass filtering (Appendix 2) which fixes the lower range at which the equipment can be used, since low frequency signals caused by small velocities are eliminated.

\section{Quantitative value}

The poor results obtained in patients with aortic stenosis seem to be related to changes in systolic waveform, an irregular, oscillatory appearance being the result of corresponding highly disturbed alterations of flow. In such a situation it is not possible to form an accurate assessment of concomitant aortic regurgitation (Fig. 7). Moreover, in associated severe aortic stenosis, disturbances of systolic flow may be prolonged into the first part of diastole.

Several other lesions of the aortic arch could cause misleading appearances but they were not encountered in this study. Coarctation of the aorta and persistent ductus arteriosus should be especially noted. Characteristic features of the blood flow in these conditions are as follows.

Coarctation of the aorta: Localised flow disturbances are found at the junction of the horizontal and descending portions of the aorta. A continuous flow component develops in this region which is also found more distally in the abdominal aorta.

Persistent ductus arteriosus: Continuous diastolic flow occurs in the aorta. Above the origin of the ductus it has the same direction as systolic flow whereas below this point, flow occurs in a reverse direction and results in a waveform comparable to that found in aortic regurgitation. Other aortopulmonary shunts may be included in this discussion but as yet we have had no experience with them.

In contrast, the results of Doppler echocardiography are highly promising if only pure aortic regurgitation is considered. Since the absence of aortic stenosis can easily be shown by echocardiography the validity of this method may be defined in each patient. The overlap between groups is undoubtedly related to the limi- 


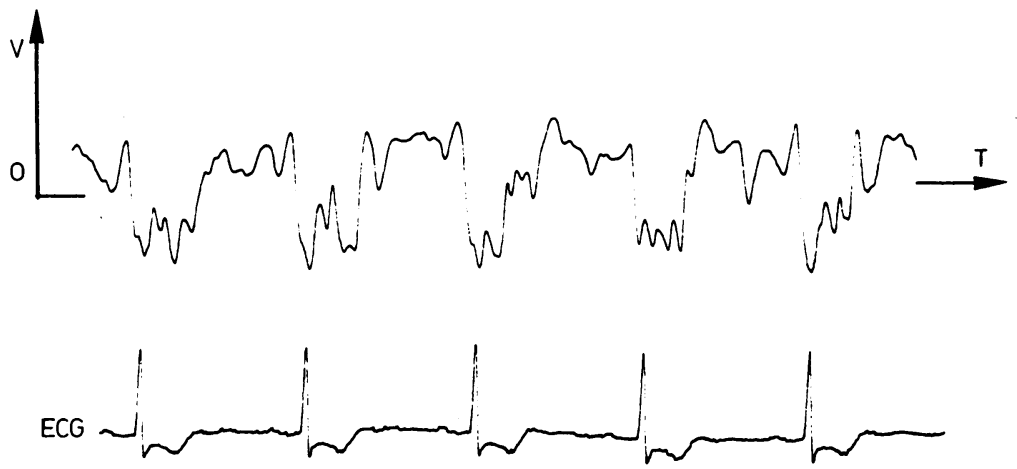

Fig. 7 Example of aortic regurgitation associated with aortic stenosis.

tations of the Doppler technique as we have already discussed, but this overlap is probably increased because of limitations of angiographic classification.

Before concluding we should mention a study ${ }^{21}$ which suggests that aortic regurgitation may be quantified by measuring aortic arch blood flow in the same region but using a continuous wave Doppler technique. As a reference method the author has used angiography to measure the regurgitation fraction and obtained a high correlation coefficient $(r=0.91)$. He drew attention, however, to the fact that this method might not be applicable to all patients. This comment demands some discussion since the equipment under consideration emits signals continuously and does not allow depth discrimination. Criteria put forward to reject carotid artery blood flow assume that electronic filtering totally suppresses the physiological component of this flow in diastole; venous flow may certainly be rejected if veins are not dilated but in the opposite situation they stay within the axis of the transducer, regardless of its angulation, and therefore prevent a proper examination. Finally the problem of severe tricuspid regurgitation giving rise to misleading results of blood flow was not considered in this study.

\section{Conclusion}

The analysis of blood flow in the aortic arch using Doppler echocardiography seems to be a valuable method for the diagnosis of aortic regurgitation. In pure aortic reflux this technique allows calculation of a ratio between maximum systolic forward velocity and end-diastolic reflux which provides a quantitative index comparable to that obtained with supravalvular aortography as used at present.

We wish to express our gratitude to Mr Alain Barbet, Mr Michel Xhaard, and Mr Jean-Paul Gugliemi for their technical assistance.

\section{APPENDIX 1}

Velocity ambiguities are induced by the sampling $\frac{\mathbb{D}}{\circ}$ procedure of the ultrasonic investigation. Sampling frequency or pulse repetition rate is the frequency at $₹$ which the ultrasound wave is sent. For a given pulse $\overrightarrow{0}$ repetition frequency, there is a maximum analysable $\stackrel{\infty}{\infty}$ depth which is linked to the time interval separating two wave trains, and a maximum measurable velocity, which is directly linked to this repetition frequency by the mathematical theories of sampling. When the measured velocity is greater than the maximum $\frac{}{\Phi}$ measurable velocity, one sees the occurrence of aliais- $\stackrel{\square}{a}$ ing: the instrument introduces an error, appearing as $\overrightarrow{0}$ a hook phenomenon, which can reach the point of inversion in the curves (Fig. 3).

\section{APPENDIX 2}

The heart is a moving structure. The displacements of $\frac{}{\circ}$ the walls are all the more easily measurable by the use 3 . of the Doppler effect, as they correspond to energy 8 rich echoes. Generally, parietal velocities are less than those of the blood cells, and therefore can be differen-음 tiated by means of high pass filters which eliminate the low frequencies.

\section{References}

1 Feigenbaum H. Echocardiography. Philadelphia: Lea \& Febiger, 1981: 275-7.

2 Diebold B, Théroux P, Bourassa MG, Peronneau P, $\stackrel{\oplus}{\mathbb{D}}$ Guermonprez JL. Non-invasive assessment of aortocoronary bypass graft patency using pulsed Doppler echocardiography. Am f Cardiol 1979; 43: 10-6.

3 Diebold B, Théroux P, Bourassa MG, et al. Non-invasive $\stackrel{\mathbb{D}}{\square}$

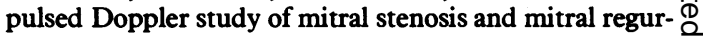
gitation: preliminary study. Br Heart f 1979; 42: 168-75. 
4 Thuillez C, Théroux P, Bourassa MG, et al. Pulsed Doppler echocardiographic study of mitral stenosis. Circulation 1980; 61: 381-7.

5 Abbasi AS, Allen MW, Decristofaro D, Ungar I. Detection and estimation of the degree of mitral regurgitation by range-gated pulsed Doppler echocardiography. Circulation 1980; 61: 143-7.

6 Blanchard D, Diebold B, Peronneau P. Non-invasive diagnosis of mitral regurgitation by Doppler echocardiography. Br Heart $\mathcal{F}$ 1981; 45: 589-93.

7 Stevenson JG, Kawabori I, Guntheroth WG. Differentiation of ventricular septal defects from mitral regurgitation by pulsed Doppler echocardiography. Circulation 1977; 56: 14-8.

8 Stevenson JG, Kawabori I, Dooley T, Guntheroth WG. Diagnosis of ventricular septal defect by pulsed Doppler echocardiography. Circulation 1978; 58: 322-6.

9 Goldberg SJ, Areias JC, Spitaels SEC, De Villeneuve VH. Use of time interval histographic output from echo-Doppler to detect left-to-right atrial shunts. Circulation 1978; 58: 147-52.

10 Stevenson JG, Kawabori I, Guntheroth WG. Noninvasive detection of pulmonary hypertension in patent ductus arteriosus by pulsed Doppler echocardiography. Circulation 1979; 60: 355-9.

11 Young JB, Quinones MA, Waggoner AD, Miller RR. Diagnosis and quantification of aortic stenosis with pulsed Doppler echocardiography. Am $\mathcal{F}$ Cardiol 1980; 45: 987-94.

12 Ciobanu M, Abbasi AS, Allen M, Hermer A, Spellberg R. Pulsed Doppler echocardiography in the diagnosis and estimation of severity of aortic insufficiency. $A m \mathcal{F}$ Cardiol 1982; 49: 339-43.

13 Veyrat C, Cholot N, Abitbol G, Kalmanson D. Noninvasive diagnosis and assessment of aortic valve disease and evaluation of aortic prosthesis function using echo pulsed Doppler velocimetry. Br Heart $\mathcal{F}$ 1980; 43: 393413.

14 Quinones MA, Young JB, Waggoner AD, Ostojic MC, Ribeiro LGT, Miller RR. Assessment of pulsed Doppler echocardiography in detection and quantification of aortic and mitral regurgitation. Br Heart $\mathcal{F} 1980 ; 44$ : 612-20.

15 Nichols WW, Pepine CJ, Conti CR, Christie LG, Feldman RL. Quantitation of aortic insufficiency using a catheter-tip velocity transducer. Circulation 1981; 64: 375-80.

16 Mennel RG, Joyner CR Jr, Thompson PD, Pyle RR, Macvaugh H III. The preoperative and operative assessment of aortic regurgitation. Am $\mathcal{F}$ Cardiol 1972; 29: 360-6.

17 Hunt D, Baxley WA, Kennedy JW, Judge TP, Williams JE, Dodge HT. Quantitative evaluation of cineaortography in the assessment of aortic regurgitation. Am $\mathcal{F}$ Cardiol 1973; 31: 696-700.

18 Linhart JW. Aortic regurgitation. Clinical, hemodynamic, surgical, and angiographic correlations. Ann Thorac Surg 1971; 11: 27-37.

19 Cohn LH, Mason DT, Ross J Jr, Morrow AG, Braunwald E. Preoperative assessment of aortic regurgitation in patients with mitral valve disease. Am $\mathcal{F}$ Cardiol 1967; 19: 177-82.

20 Frank MJ, Casanegra P, Nadimi M, Migliori AJ, Levinson GE. Measurement of aortic regurgitation by upstream sampling with continuous infusion of indicator. Circulation 1966; 33: 545-57.

21 Boughner DR. Assessment of aortic insufficiency by transcutaneous Doppler ultrasound. Circulation 1975; 52: 874-9.

Requests for reprints to Professor J L Guermonprez, Hôpital Broussais, 96 rue Didot, 75014 Paris, France. 